\title{
Erratum: Laser-stimulated deexcitation of Rydberg antihydrogen atoms [Phys. Rev. A 99, 013418 (2019)]
}

\author{
D. Comparat (1) and C. Malbrunot 10
}

(Received 20 December 2019; published 30 January 2020)

DOI: 10.1103/PhysRevA.101.019904

In our original paper, we presented a method to optimally mix several states within a set of $n$ manifolds $(n=20-30)$ in antihydrogen. The main purpose was to couple high angular momentum states to low ones using well-chosen electricand magnetic-field magnitudes and orientation in order to be able to drive rapidly the population down to low-lying states [which then decay spontaneously to the ground state (GS)] using a broadband laser.

In our original paper, we treated the individual levels of the several excited $n$ manifolds (containing $n^{2}$ levels each) independently, meaning that we neglected repopulation of the considered levels by other states. This is, however, not a valid assumption especially for a high power laser. We correct, here, this fact which leads to the main modification that the deexcitation time is not only limited by the decay time of the lower manifold to which the $n=20-30$ population is driven to, but also by the number of states addressed as we showed in Ref. [1].

This point can be illustrated using the simple model of fully mixed $l, m$ states (see Sec. II in our original paper).

For ease of exposition, we recall, in Fig. 1 (with additional nomenclature), the first figure of the original paper.

Under perfect mixing, all states behave in a similar manner as the $\left|\psi_{n}\right\rangle \approx \sum_{l m} \frac{1}{n}|n l m\rangle$ mixed state coupled to all states of the lower $n^{\prime}$ manifold, the number of which is $n_{3}=n^{\prime 2}$. With such a hypothesis, we obtain the corrected rate equations for the population $N_{i}$ of individual levels,

$$
\begin{aligned}
n_{2} \frac{d N_{2}}{d t} & =-n_{2} \Gamma_{\text {stim }}\left(N_{2}-N_{3}\right)-n_{2} \Gamma_{\text {photo }} N_{2}, \\
n_{3} \frac{d N_{3}}{d t} & =n_{2} \Gamma_{\text {stim }}\left(N_{2}-N_{3}\right)-n_{3} \Gamma_{\text {spon }} N_{3}, \\
\frac{d N_{4}}{d t} & =n_{3} \Gamma_{\text {spon }} N_{3},
\end{aligned}
$$

where $\Gamma_{\text {photo }}, \Gamma_{\text {stim }}$, and $\Gamma_{\text {spon }}$ are the averaged photoionization, stimulated, and spontaneous rates respectively. We found, in our original paper, the following main relations for the $n$ and $n^{\prime}$ manifolds:

Published by the American Physical Society under the terms of the Creative Commons Attribution 4.0 International license. Further distribution of this work must maintain attribution to the author(s) and the published article's title, journal citation, and DOI.
(1) $\Gamma_{\text {spon }} \approx 1.54 \times 10^{10} \mathrm{~s}^{-1} \times n^{\prime-4.5}$ which is the average decay rate of the $n^{\prime}$ manifold.

(2) $\Gamma_{\text {stim }} \approx 0.2 \mathrm{~s}^{-1} \frac{I}{1 \mathrm{~W} / \mathrm{m}^{2}} \frac{2 \pi \times 5000 \mathrm{GHz}}{\Gamma_{\mathrm{L}}} n^{5.2} n^{-5}$ for an unpolarized laser of intensity $I$ and spectrally Lorentzian FWHM $\Gamma_{\mathrm{L}}$ linewidth.

(3) $\frac{\Gamma_{\text {stim }}}{\Gamma_{\text {photo }}} \approx 630 n^{\prime-2.9}$. For a low $n^{\prime}$ value $\left(n^{\prime}=2,3\right)$, the photoionization rate $\Gamma_{\text {photo }}$ can, thus, be neglected.

When coupling an initial population of $n=20-30$ Rydberg states the equations are similar except that $n_{2}=\sum_{n=20}^{n=30} n^{2} \approx$ 7000 and $\Gamma_{\text {stim }}$ have to be averaged over $n=20-30$ to become $\Gamma_{\text {stim }} \approx 8 \times 10^{-6} \mathrm{~s}^{-1} \frac{I}{1 \mathrm{~W} / \mathrm{m}^{2}} \frac{2 \pi \times 5000 \mathrm{GHz}}{\Gamma_{\mathrm{L}}}\left(\frac{n^{\prime}}{3}\right)^{5.2}$.

The saturation occurs when $n_{2} \Gamma_{\text {stim }}>n_{3} \Gamma_{\text {spon }}$. In that case, all levels become equipopulated (so $N_{3} \approx N_{2}$ ), thus, the initial population of $N$ atoms distributed over the $n_{2}$ levels gets spread over the $n_{2}+n_{3}$ levels and decays to populate the ground state at a rate $\Gamma_{\text {deexcitation }}=\Gamma_{\text {spon }} \frac{n_{3}}{n_{2}+n_{3}} \approx \Gamma_{\text {spon }} \frac{n_{3}}{n_{2}}$.

Thus, the main correction to the original paper is that the ground state will not be populated in a 100-ns timescale but rather at a maximum rate (for $n^{\prime}=3$ ) of $\Gamma_{\text {deexcitation }} \approx \frac{1}{7 \mu \mathrm{s}}$. The saturation will occur for $I>1.8 \times 10^{10} \mathrm{~W} / \mathrm{m}^{2}$ which corresponds to $\sim 10 \mathrm{~J}$ during $7 \mu$ s if focused on $1 \mathrm{~cm}^{2}$.

This simple model assumed a steady equipopulation, whereas, in fact, dipole transitions between different states are different, and optical pumping will occur in a more complex manner. In order to verify these simple results, we solved numerically the set of rate equations for all mixed $\left(n, m_{1}, m_{2}\right)$ levels as calculated at first order in our original paper with $20 \leqslant n \leqslant 30$ under the presence of the optimum electric- and magnetic-field values found in the original paper and implemented laser-stimulated rates to the $n^{\prime}=3$ manifold that then decay spontaneously to the ground state. The results are given in Fig. 2. For a laser power of $5 \times 10^{9} \mathrm{~W} / \mathrm{m}^{2}, \sim 60 \%$ of the atoms with an initial statistical distribution in the $20 \leqslant n \leqslant 30$ manifolds are brought to the ground state within $10 \mu \mathrm{s}$. This is in good agreement with the considerations above but fortunately with a slightly lower required power. As noted in our original paper [Fig. 4(a)], a more refined nonperturbative calculation of the coupling between $n$ states due to the $\boldsymbol{E}$ and $\boldsymbol{B}$ fields tends to reduce the necessary laser power needed. Therefore, $1 \mathrm{~J}$ during $10 \mu \mathrm{s}$, that is, $100 \mathrm{~kW} / \mathrm{cm}^{2}$ should be sufficient to achieve an efficient deexcitation. This could even relax the requirements for the laser system found in the original paper. 


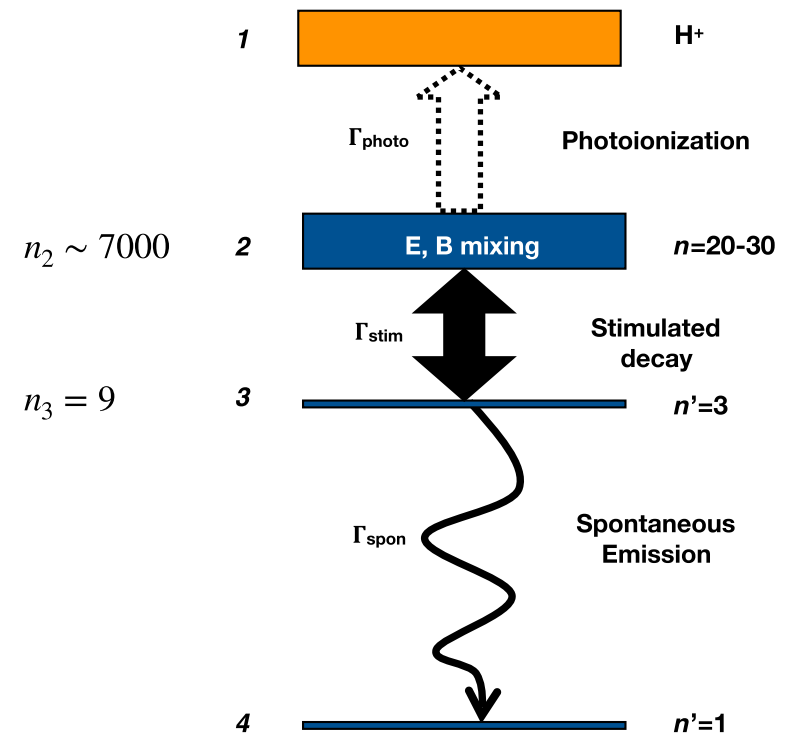

FIG. 1. Principle of the stimulated decay of Rydberg antihydrogen atoms. Combined electric $\boldsymbol{E}$ and magnetic $\boldsymbol{B}$ fields mix the $n=20-30$ Rydberg states (number of states $n_{2}$ ) that can then be stimulated down to $n^{\prime}$ (number of states $n_{3}=n^{\prime 2}$ ) at a rate $\Gamma_{\text {stim. }}$. The states within the $n^{\prime}=3$ manifold will quickly decay toward the ground state. The competing photoionization mechanism is also shown.

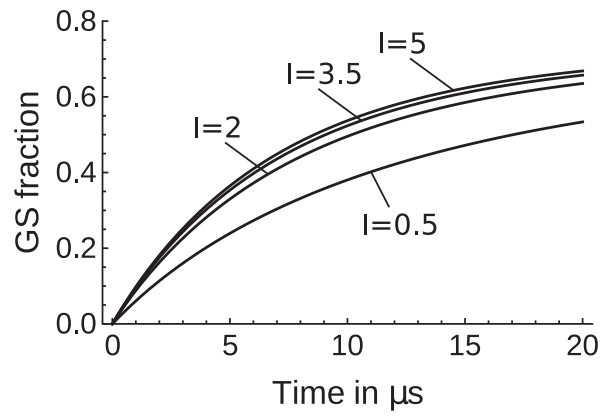

FIG. 2. Ground-state (GS) fraction as a function of time for a population initiated with a statistical distribution in the $n=20-30$ manifolds. The states are mixed by the optimal $\boldsymbol{E}$ and $\boldsymbol{B}$ fields found in our original paper. A laser light with $\lambda=832 \mathrm{~nm}, \Gamma_{L}=$ $5000 \mathrm{GHz}$, and different intensities drives the population down to the $n^{\prime}=3$ states which, subsequently, decay to the GS. The light intensities (in units of $\mathrm{GW} / \mathrm{m}^{2}$ ) are indicated in the graph.

In conclusion, the microsecond timescale deexcitation can still be considered pulsed for many applications, and, thus, the main point of the original paper which was to allow rapid and efficient deexcitation via a laser remains valid.

[1] T. Wolz, C. Malbrunot, M. Vieille-Grosjean, and D. Comparat, Stimulated decay and formation of antihydrogen atoms, arXiv:1912.03163. 